\title{
Study on modes of axial flow Pump Blade based on Fluid-structure Coupling
}

\author{
Zhang Yu \\ School of Mechanical Science and Engineering \\ Huazhong University of Science and Technology \\ Wuhan, China, 027-87557664 \\ zhyu1003@gmail.com
}

\section{Li Shan De}

School of Mechanical Science and Engineering Huazhong University of Science and Technology Wuhan, China, 027-87557664

lishande@gmail.com

\author{
Huang Qi Bai* \\ School of Mechanical Science and Engineering \\ Huazhong University of Science and Technology \\ Wuhan, China, 027-87557664 \\ *Corresponding author: qbhuang@mail.hust.edu.cn
}

Liu Yan Jun

Catic Industrial Wuhan Aviation Instrument Co., LTD Wuhan, China, 0717-3381499

lllflying@yahoo.cn

\begin{abstract}
The determination of frequencies and models in fluid of the structure is an important problem in the fluid-solid coupling study. We analyzed an axial flow pump blade by finite element analysis software ADINA in the paper, then we got its models in air and fluid respectively. The modal frequencies of different orders of the structure in fluid decreased in different degrees compared with frequencies in air.
\end{abstract}

Keywords-ADINA; fluid-solid coupling; models in fluid

\section{INTRODUCTION}

The natural vibration characteristics of structure in water should be considered according to the need of engineering design. Initially, someone put forward that we can solve the natural vibration characteristics of structure in air using the theory method, then multiply the results with a reduction coefficient according to the experience to get the natural vibration frequencies in water. This method will produce great error, because the decreased degree of the structural frequency in water is related to many factors. So multiplying a coefficient simply is not reasonable. With the development of dynamics and calculation methods, fluid-structure coupling shows its unique superiority gradually in solving the above problem. For almost a century Scholars at home and abroad studied on fluid-structure dynamic coupling theory and method, and have obtained some achievements. However the theory and practice have not be unified because of the complexity of fluid-structure coupling.

The vibration of the structure in the fluid can lead the vibration of the fluid around, conversely the movement of fluid would influent the stress on the structure, that the fluid-structure coupling problem comes into being ${ }^{[2][3][4][5]}$. The determination of frequencies and models in fluid of the structure is an important problem in the fluid-solid coupling study ${ }^{[6]}$.In the paper, we analyzed the fluid-structure coupling models of the structure in fluid using the weak

Financial support from the National Nature Science Foundation of China (No. 51175195) is gratefully acknowledged. coupling method by CAE software ADINA.

\section{THE FluID-STRUCTURE COUPLING THEORY}

Assuming that the fluid is ideal flow, which is uniform, inviscid and irrotational. Now consider the additional hydrodynamic pressure induced by the vibration of the structure. The kinetic equation of the structure

$$
M r+C r+K r=F_{p}+F
$$

Where $M$ is the mass matrix of structure; $C$ is the damping matrix of structure; $K$ is the stiffness matrix of structure; $F_{p}$ is the fluid force on the fluid-structure interface induced by the vibration of the structure; $F$ is the external excitation except for $F_{p} ; r$ is the displacement vector of structure.

The locations of the fluid notes on the fluid-structure interface are determined by the kinematic conditions. The displacements of other notes are determined by the program automatically to keep the quality of the initial mesh. In the static analysis, even the fluid note's displacement changes, its speed is also considered to be zero, the speed of fluid on the fluid-structure interface is zero.

According to the kinetic conditions, we can turn the distributed force of the flow on the fluid-structure interface to concentrated force by formula (2), and then apply it to the structure note

$$
F(t)=\int h^{d} \tau_{f} \cdot d S
$$

Where $h^{d}$ is the displacement of structure notes.

\section{CALCULATION MODEL}

The entity is an axial flow pump blade, and the airfoil is 
Gottingen 490.The elastic modulus of the material is $E=2.1 \times 10^{11} P_{a}$.The Poisson's ratio is 0.3.The density is $7.8 \times 10^{3} \mathrm{Kg} / \mathrm{m}^{3}$.The fluid is water, and it's bulk modulus is $E=2.3 \times 10^{9} P_{a}$, it's density is $1.0 \times 10^{3} \mathrm{Kg} / \mathrm{m}^{3}$.The viscosity of water is ignored.

When meshing, the element of 3D-solid was used for the structure, and the element of 3D-fluid was used for the fluid. After setting up the face-link, element group and mesh density, we could begin to mesh the model. The grid on boundary could link up, although we meshed different element into different number of notes.

The installation surface was restrained fully to simulate the blade's installation state. The blade's grid model is depicted in figure 1.

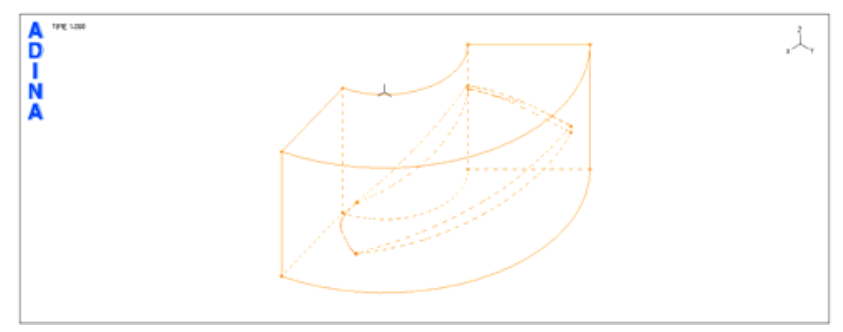

Figure 1. the blade's grid model

The structure model of the blade in water is depicted in figure 2, and the grid model of the fluid-structure coupling region is depicted in figure 3 .

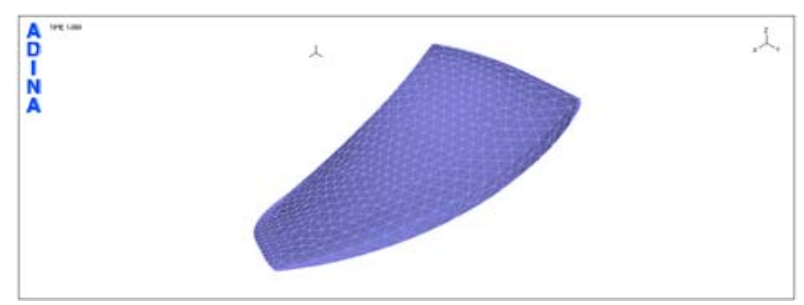

Figure 2. the structure mod el of the blade in water

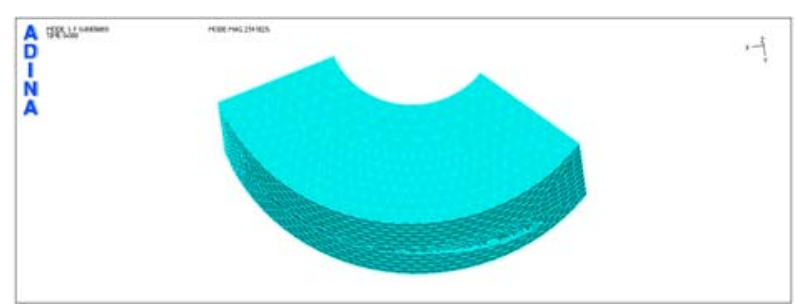

Figure 3. the grid model of the fluid-structure coupling region

\section{RESUlTS AND ANALYSIS}

Firstly, we calculated models of the structure in air, then taking the fluid model into account, we calculated the coupling frequencies by fluid-structure coupling method. The results of calculation are depicted in table 1. $f_{w 1}$ and $f$ represent the natural frequencies considering the fluid-structure coupling or not respectively.
Table I. THE COMPARISON OF THE MODAL FREQUENCIES

\begin{tabular}{|c|c|c|c|c|}
\hline order & 1 & 2 & 3 & 4 \\
\hline$f(H z)$ & 1804 & 2451 & 3750 & 4995 \\
\hline$f_{w 1}(H z)$ & 1191 & 1859 & 2947 & 3088 \\
\hline$\left(f-f_{w 1}\right) / f \times 100 \%$ & $33.98 \%$ & $24.15 \%$ & $21.41 \%$ & $38.18 \%$ \\
\hline
\end{tabular}

It can be seen from the data in table 1, that the modal frequencies in different orders decrease in different degrees. The influence of water on natural frequencies is mainly caused by doing negative work in the direction of vibration, and the works on different modes of vibration are not equal. So it's not accurate to get the structure modal in water by multiplied the frequencies in air with a coefficient according to the experience.

The blade's modal shapes of the top four orders in air and in water are depicted in figure 4 and figure 5 respectively.

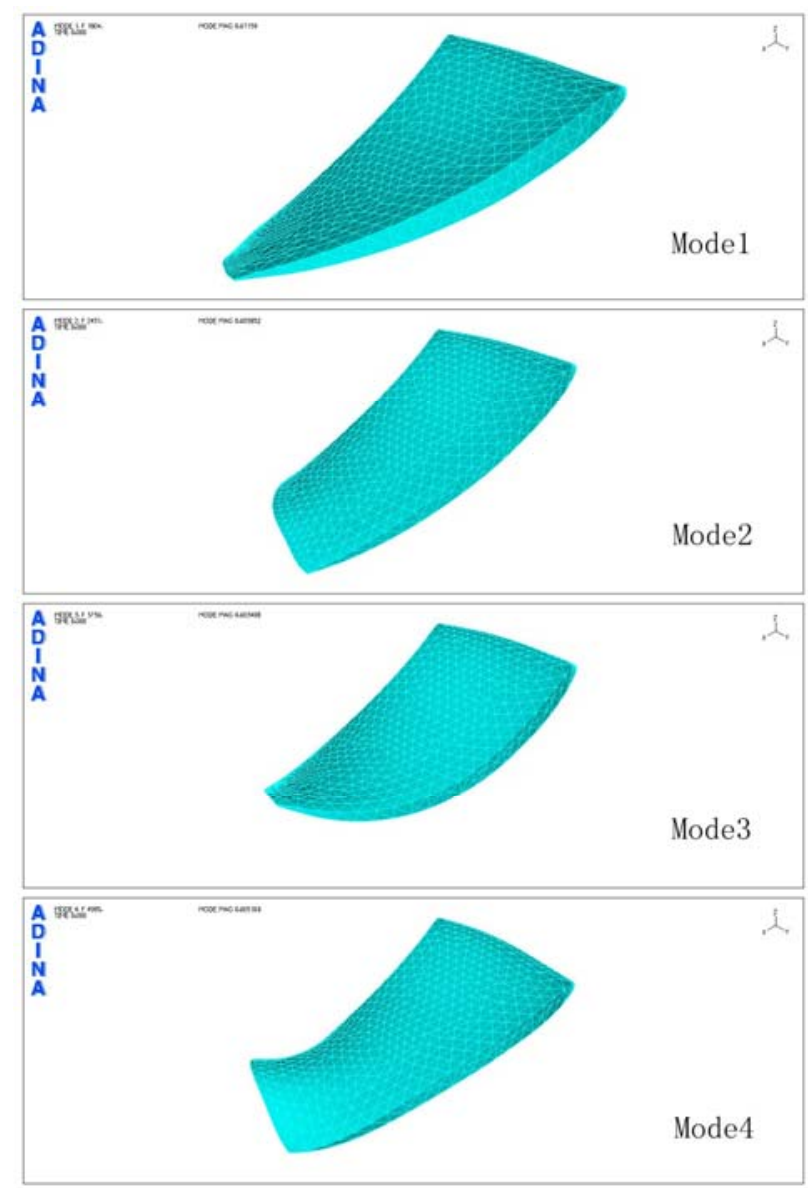

Figure 4. the blade's modal shapes of the top four orders in air 


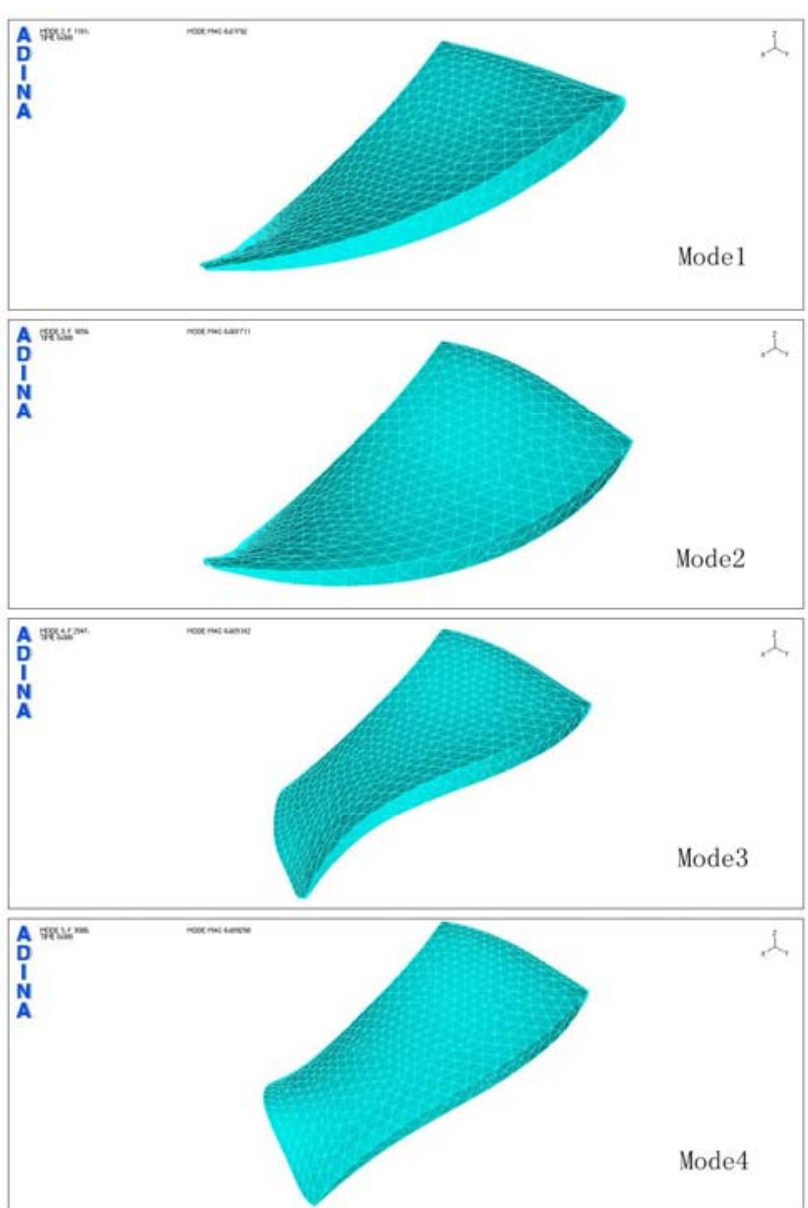

Figure 5. the blade's modal shapes of the top four orders in water

What the results show is that, compared with the mode in air, not only the modal frequencies in different orders in water decrease in different degrees, but also the blade's vibration modes in some orders in water are different from that in air. For the nonlinear structure such as the blade, on the one hand the quality of the water will affect the mass matrix of the structure, on the other hand the pressure of the water will affect the stiffness matrix of the structure, so not only the quality of the water but also the pressure of it could affect the natural frequencies of the structure. What's more, the shape and size of the water model could also affect the vibration modes of the blade in water, because not only the water's static pressure but also its dynamic pressure was taken into account during the fluid-structure coupling calculation process by ADINA.

\section{CONCLUSION}

Through the analysis above, we have calculated the blade's modes in air and water respectively. The results show that compared with the mode in air, the modal frequencies of the structure in different orders in water decrease in different degrees. It is necessary to avoid these frequencies in the practical application in order to prevent resonance.

\section{REFERENCES}

[1] M. Yue Ge, The Advanced Applications of Fluid and Fluid-Structure Coupling Functions in ADINA. Beijing : Chinese Traffic Publishing House, 2010.

[2] J. Li Haibin, Feng Guotai, Review on Multiphysics Coupling Analysis in Turbomachinery Design. Aviation Engine Translation.2002,2:51-56.

[3] J. Liu Qingmao, Model Test Investigation of Complex Body Structure in Water. Missiles and Space Vehicles. 1997, 228(4):23-29.

[4] M. Thomson W T, Dahleh M D. Theory of Vibration With Applications. 5th Ed. New Jersey: Prentice Hall, 1997, 183-187.

[5] M. Robert D. Blenvis Ph D. Formulas for Natural Frequency and Mode Shape. Malabar Florida: Krieger Publishing Company, 2001, 391-399.

[6] J. Huang Y Y, Orthogonality of Wet Modes in Coupled Vibrations of Cylindrical Shells Containing Liquids. Journal of Sound and Vibration.1991,145(1):51-60. 